\title{
Evolutionary Acceleration and Divergence in Procolobus kirkii
}

\author{
Katarzyna Nowak • Andrea Cardini • Sarah Elton
}

Published online: 8 November 2008

(C) Springer Science + Business Media, LLC 2008

\section{Erratum to: Int J Primatol \\ DOI 10.1007/s10764-008-9306-1}

Due to a publication error, some of the data in Table I were not displayed correctly. We publish the corrected version of the table below:

The online version of the original article can be found at http://dx.doi.org/10.1007/s10764-008-9306-1.

K. Nowak

Wildlife Conservation Research Unit (WildCRU), University of Oxford, Tubney House, Abingdon Road, Abingdon OX13 5QL, UK e-mail: katarzyna.nowak@zoo.ox.ac.uk

\section{A. Cardini}

Dipartimento del Museo di Paleobiologia e dell'Orto Botanico, Universitá di Modena e Reggio Emilia, 41100 Modena, Italy e-mail: alcardini@interfree.it

A. Cardini $\cdot$ S. Elton $(\bowtie)$

Hull York Medical School, The University of Hull, Hull HU6 7RX, UK e-mail: Sarah.Elton@hyms.ac.uk 
Table I Taxa used in analysis and sample sizes

\begin{tabular}{|c|c|c|c|c|}
\hline \multirow[t]{2}{*}{ Genus/subgenus } & \multirow[t]{2}{*}{ Species } & \multirow[t]{2}{*}{ Subspecies } & \multicolumn{2}{|l|}{$N$} \\
\hline & & & Females & Males \\
\hline \multirow{14}{*}{$\begin{array}{l}\text { Procolobus } \\
\text { (Piliocolobus) }\end{array}$} & kirkii & - & 33 & 10 \\
\hline & \multirow[t]{3}{*}{ badius } & badius & 36 & 23 \\
\hline & & temminckii & 11 & 4 \\
\hline & & waldroni & 15 & 6 \\
\hline & gordonorum & & 4 & - \\
\hline & \multirow[t]{3}{*}{ pennantii } & bouvieri & 1 & - \\
\hline & & epieni & - & 1 \\
\hline & & preussi & 31 & 10 \\
\hline & rufomitratus & - & 5 & 1 \\
\hline & \multirow[t]{5}{*}{ sp. } & ellioti & 16 & 18 \\
\hline & & foai & 3 & 6 \\
\hline & & oustaleti & 9 & 5 \\
\hline & & tephrosceles & 7 & 16 \\
\hline & & tholloni & 4 & 2 \\
\hline $\begin{array}{l}\text { Procolobus } \\
\text { (Procolobus) }\end{array}$ & verus & - & 20 & 7 \\
\hline \multirow[t]{3}{*}{ Colobus } & angolensis & - & 4 & 4 \\
\hline & guereza & - & 20 & 19 \\
\hline & polykomos & - & 11 & 7 \\
\hline
\end{tabular}

